\title{
Comparison of Calculated and Experimental Results of Fragmenting Cylinder Experiments
}

\author{
L.T. Wilson, D.R. Reedal ${ }^{a}$, M.E. Kipp, R. R. Martinez ${ }^{b}$, D.E. Grady ${ }^{\mathrm{c}}$ \\ ${ }^{2}$ Dahlgren Division Naval Surface Warfare Center \\ 17320 Dahlgren Rd, Dahlgren VA, 22448-5100 \\ 'Sandia National Laboratories, \\ Albuquerque NM, 87185-0820 \\ 'Applied Research Associates \\ 4300 San Mateo Blvd. NE Suite A-220, Albuquerque, NM 87110
}

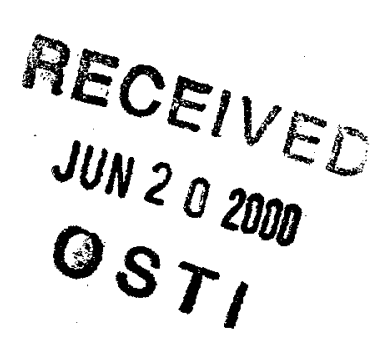

The Grady-Kipp fragmentation model provides a physically based method for determining the fracture and breakup of materials under high loading rates. Recently, this model has been implemented into the CTH Shock Physics Code and has been used to simulate several published experiments. Materials studied in this paper are AerMet 100 steel and a $90 \%$ tungsten alloy. The experimental geometry consists of a right circular cylinder filled with an explosive main charge that is initiated at its center. The sudden expansion of the resulting detonation products causes fracture of the cylinder. Strain rates seen in the cylinder are on the order of $10^{4} \mathrm{~s}^{-1}$. The average fragment sizes calculated with the Grady-Kipp fragmentation model successfully replicate the mean fragment size obtained from the experimental fragment distribution. When Poisson statistics are applied to the calculated local average fragment sizes, good correlation is also observed with the shape of the experimental cumulative fragment distribution. The experimental fragmentation results, CTH numerical simulations, and correlation of these numerical results with the experimental data are described.

\section{INTRODUCTION}

The fragment formation accompanying expansion of a cylinder that has been accelerated with explosives has been discussed in many places (e.g., Mock and Holt, 1983; Mott, 1943). Much work has been invested in understanding the fragmentation process and predicting fragment characteristics that ensue. In the present study, fragmentation of two materials - a high-strength steel alloy and a tungsten alloy - is examined under conditions of an expanding cylindrical geometry, accelerated by an explosive charge.

Numerical simulations of the devices were made with the CTH Eulerian shock wave propagation code (McGlaun, et al., 1990). This multi-dimensional shock physics Eulerian code is capable of modeling dynamic events that include explosive detonation and high velocity impact. The CTH code solves the differential equations describing conservation of mass, momentum and energy during transient dynamic events on a fixed spatial mesh. CTH is capable of tracking the interactions of up to 20 materials. This code contains models suitable to describe material response under most conditions encountered in shock physics, including the explosives and inert solids for the current application. A variety of material insert geometries facilitate the modeling of complex devices. The Eulerian structure of the code permits large deformations associated with explosive or impact events to be accommodated. The characteristic fragment dimensions in the numerical simulations are determined principally by the strain rate, $\dot{\varepsilon}$, at the time of fracture. The basic relationships between the strain rate and the average fragment dimensions that result as materials fracture under high strain rate loading conditions have been derived by Grady (1988). In the current analyses, the temperature and strain rate regimes are such that the 


\section{DISCLAIMER}

This report was prepared as an account of work sponsored by an agency of the United States Government. Neither the United States Government nor any agency thereof, nor any of their employees, make any warranty, express or implied, or assumes any legal liability or responsibility for the accuracy, completeness, or usefulness of any information, apparatus, product, or process disclosed, or represents that its use would not infringe privately owned rights. Reference herein to any specific commercial product, process, or service by trade name, trademark, manufacturer, or otherwise does not necessarily constitute or imply its endorsement, recommendation, or favoring by the United States Government or any agency thereof. The views and opinions of authors expressed herein do not necessarily state or reflect those of the United States Government or any agency thereof. 


\section{DISCLAIMER}

Portions of this document may be illegible in electronic image products. Images are produced from the best available original document. 
fragmentation is governed by the fragmentation toughness, $K_{F}$, of the materials. The average fragment size, $\mathrm{S}$, is determined from

$$
S=\left(\frac{\sqrt{24} K_{F}}{\rho c \dot{\varepsilon}}\right)^{2 / 3}
$$

where $\rho$ is the density, and $c$ is the sound speed (Grady, 1988). This fragment information is included in the simulation, but does not couple back into the calculation to form discrete fragments (Kipp, et al., 1993). The calculation is triggered when the Johnson-Cook failure model, used as a 2-parameter pressure dependent strain to failure model, indicates that the material has reached failure (Johnson and Cook, 1985). Currently, the numerical simulation models material fracture by introducing void into a cell to effect the unloading from a tensile state to a state of zero stress. As was shown by Grady and Benson (1983) and Kipp and Grady (1986), a uniform strain rate leads to a single average fragment size, but an essentially Poisson distribution of sizes about that mean. This principle is applied here to the local average sizes calculated by the code to obtain a final statistical fragment distribution.

\section{DESCRIPTION OF EXPERIMENTS}

The cylinder expansion experiments described here are part of a continuing series of tests designed to quantify the fragmentation characteristics of high strength metals. The results of the tungsten alloy test were previously reported by Rice, et al., (1996). The test results of the AerMet 100 material are from an ongoing experimental program and are not yet documented. The objectives of these tests are to study the cylinder expansion prior to and including case rupture, quantify the initial fragment velocity, and determine the fragment mass distribution.

The test hardware consisted of a $90 \%$ tungsten alloy cylinder and a heat-treated AerMet 100 steel cylinder. Each cylinder was $20.3 \mathrm{~cm}$ in length and had a $20.3 \mathrm{~cm}$ inner diameter. The charge to mass ratio was chosen to be near unity. The main charge explosive, PBXN-110, was center initiated with a $\mathrm{CH}-6$ booster. Both units were confined with steel end plates. A sketch of the test units is shown in Figure 1. Specific dimensions for each test unit are located in Table 1.

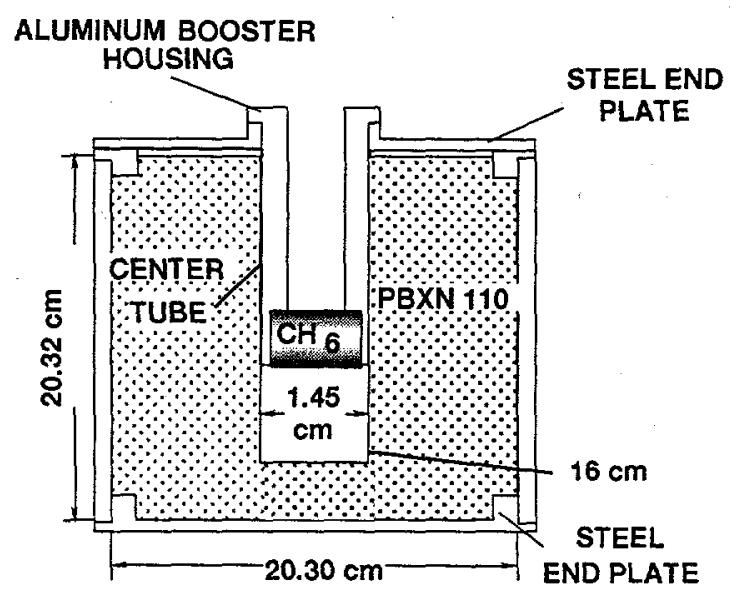

Figure 1. Hardware Geometry

Table 1.

Specific Dimensions for Each Test Unit

\begin{tabular}{lcc}
\hline & $\begin{array}{c}\mathbf{9 0 \%} \\
\text { Tungsten } \\
\text { Alloy }\end{array}$ & $\begin{array}{c}\text { AerMet } \\
\mathbf{1 0 0} \\
\text { Steel }\end{array}$ \\
\hline Density & $17230 \mathrm{~kg} / \mathrm{m}^{3}$ & $7940 \mathrm{~kg} / \mathrm{m}^{3}$ \\
Inner Diameter & $20.24 \mathrm{~cm}$ & $20.32 \mathrm{~cm}$ \\
Outer Diameter & $21.02 \mathrm{~cm}$ & $21.97 \mathrm{~cm}$ \\
Wall Thickness & $0.391 \mathrm{~cm}$ & $0.82 \mathrm{~cm}$ \\
Cylinder Mass & $8.87 \mathrm{~kg}$ & $8.85 \mathrm{~kg}$ \\
Explosive Mass & $9.27 \mathrm{~kg}$ & $10.15 \mathrm{~kg}$ \\
\hline
\end{tabular}

The test setup included high-speed framing camera, flash radiography, and soft fragment recovery. The high-speed framing camera recorded case expansion and onset of fracture. The flash radiography provided fragment velocity and polar ejection angle distribution. The soft fragment recovery was used to collect fragments in order to determine fragment mass distribution. The fragments were collected from a $25^{\circ}$ azimuthal sector of the 
cylinder. A sketch of the test setup is provided in Figure 2.

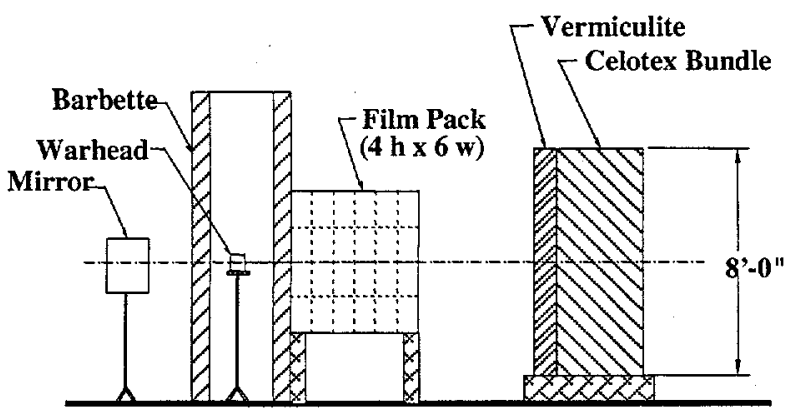

Figure 2. Test Setup

\section{NUMERICAL SIMULATIONS AND CORRESPONDING TEST DATA}

The axisymmetric simulations had a uniform numerical resolution of $0.5 \mathrm{~mm}$. The explosive parameters for the PETN booster were for a density of $1.77 \mathrm{~g} / \mathrm{cm}^{3}$, with a detonation velocity of $8.3 \mathrm{~km} / \mathrm{s}$ as defined by Dobratz and Crawford, (1985). The main charge used for both the $90 \%$ tungsten alloy and AerMet 100 steel experiments was PBXN-110, represented here with either the HMX explosive PBX-9404 or Composition B3, both simulated with a reactive burn model. The cylinder walls were modeled assuming an elastic perfectly plastic constitutive response (where the plastic flow stresses were obtained from split Hopkinson pressure bar measurements) and Mie Gruneisen equations of state (LANL, 1969). In the current CTH implementation, the fracture model uses the Johnson-Cook failure model (Johnson and Cook, 1985) as a 2-parameter pressure dependent strain to failure model. The two damage parameters needed for our calculations were estimated using a combination of experimental results and handbook values for spall stress, strain to failure, and quasi-static fracture stress. The needed $\mathrm{K}_{\mathrm{F}}$ parameter of Equation 1 was estimated using the results of the two experiments.

\subsection{The 90\% Tungsten Alloy Experiment}

The cross-section of the axisymmetric numerical representation of the tungsten experiment, and corresponding framing camera results, are shown in Figures 3 through 5 . In the CTH representations, pressure contours are on the left, material plots are on the right. The detonation front radiates as a spherical wave, first reaching the axial center of the tungsten cylinder wall at about $13 \mu \mathrm{s}$. This central region of the cylinder experiences the first radial outward motion. As the curved front of the detonation wave expands along the cylinder wall, the wall assumes the curved shape seen in Figures 4 and 5. The complex shocks that develop in the explosive can also be seen in the color pressure contours, shown on the left side of the CTH images in Figures 4 and 5. The numerical and experimental results correlate well.
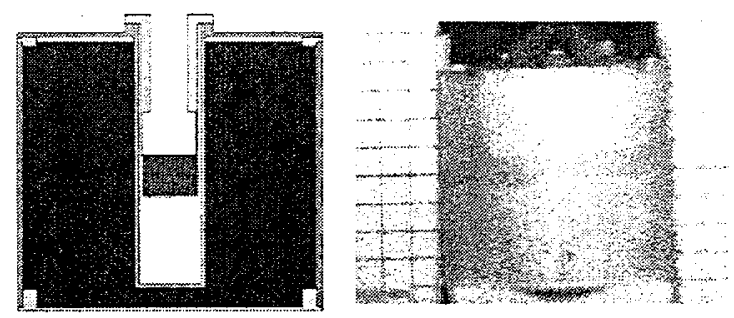

Figure 3. Comparison of $\mathrm{CTH}$ Calculation and 90\% Tungsten Alloy Cylinder at $\mathrm{T}=0 \mu \mathrm{s}$
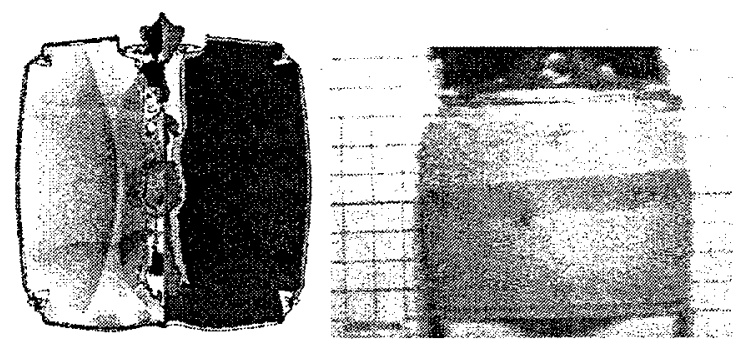

Figure 4. Comparison of CTH Calculation and 90\% Tungsten Alloy Cylinder at $\mathrm{T}=25 \mu \mathrm{s}$ 

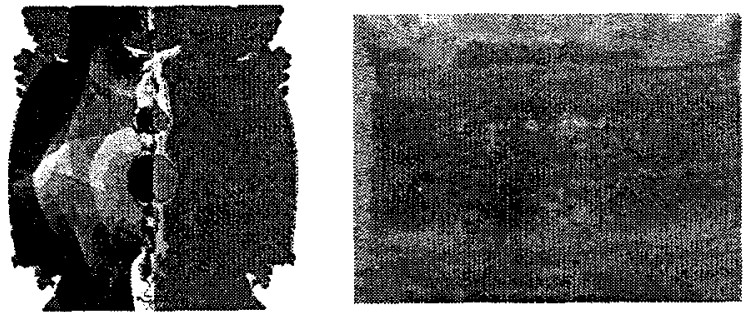

Figure 5. Comparison of CTH Calculation and 90\% Tungsten Alloy Cylinder at $\mathrm{T}=50 \mu \mathrm{s}$

As the $90 \%$ tungsten alloy cylinder expands, the largest strain is in the circumferential direction. Data from the cylinder test indicate a strain to fracture of $9 \%$. The expansion velocity at that time determines the strain rate, which is maintained at nominally $1.4 \times 10^{4} / \mathrm{s}$ over a fairly large range of strain (see Figure 6).

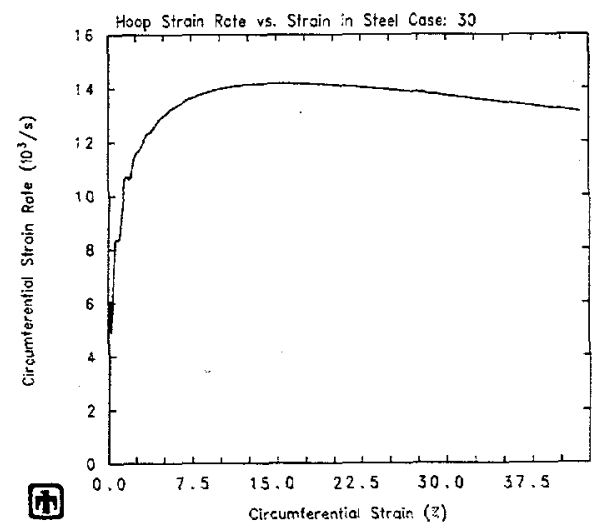

Figure 6. Typical Strain Rate Vs. Circumferential Strain History in the Tungsten (Monitored at the Axial Center of Cylinder)

As noted previously, the fragment characteristic dimensions are controlled largely by the strain rate at the time of fracture, and the fracture toughness of the metal. In this case, the tungsten has a toughness of about $30 \mathrm{MPa} \mathrm{m}^{1 / 2}$, which corresponds to an average fragment dimension of nearly $3 \mathrm{~mm}$. Assuming cubical fragments, this provides an average mass of about $0.4 \mathrm{~g}$ (6.2 grains), compared with the experimental average mass of ( 9 grains). The numerical simulation indicates that $99 \%$ of the tungsten has fragmented. However, the circumferential strain rates tend to be larger than the axial strain rates in most regions of the cylinder, and the axial strain to failure occurs later than that in the circumferential direction. For example, in the center of the cylinder, the axial strain rate is about $6 \times 10^{3} / \mathrm{s}$, which corresponds to an average fragment size of about $5 \mathrm{~mm}$, larger than the circumferential average size and giving the fragment an aspect ratio of about 1.67 (Note the fragments shown in Figure 7). In addition, the thickness of the cylindrical wall at the time of fracture should be less than $3 \mathrm{~mm}$, leading to an approximate fragment mass of about $0.7 \mathrm{~g}$ (11 grains), which is slightly larger than the average collected in the experiment.

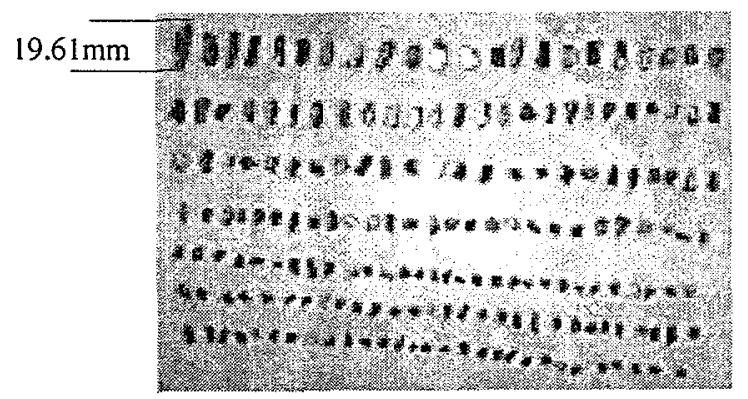

Figure 7. Fragments Recovered from the 90\% Tungsten Alloy Test

Unlike the actual collected fragments, calculated fragment size distributions are constructed on the assumption that the fragments are cubical, as determined from the local circumferential strain rates. The fragment sizes from all the masses of the tungsten are assembled into these mass and number distributions (Figure 8). The fragments are partitioned into bins of size $0.2 \mathrm{~mm}$. The preponderance of the mass clearly falls near a size of $3 \mathrm{~mm}$, as discussed, with a significant number of fragments also in the $1 \mathrm{~mm}$ size region. In these figures, the data plotted in the bins are raw local averages from the numerical simulation. 

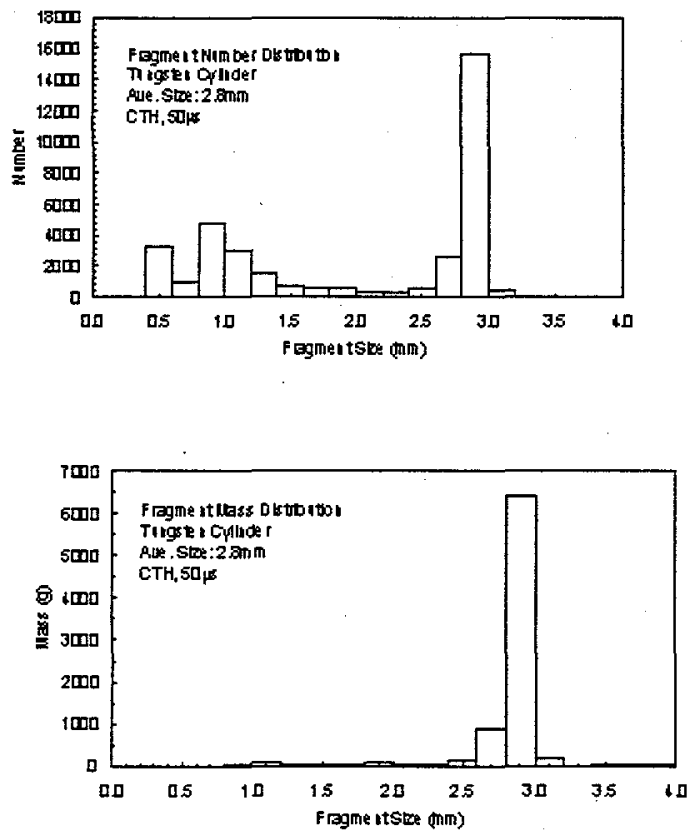

Figure 8. Fragment Number (Top) and Mass (Bottom) Distributions for the 90\% Tungsten Alloy Cylinder (Fragment Bin Size: $0.2 \mathrm{~mm}$ )

The cumulative mass distribution based upon these results is compared to the experimental data in Figure 9.

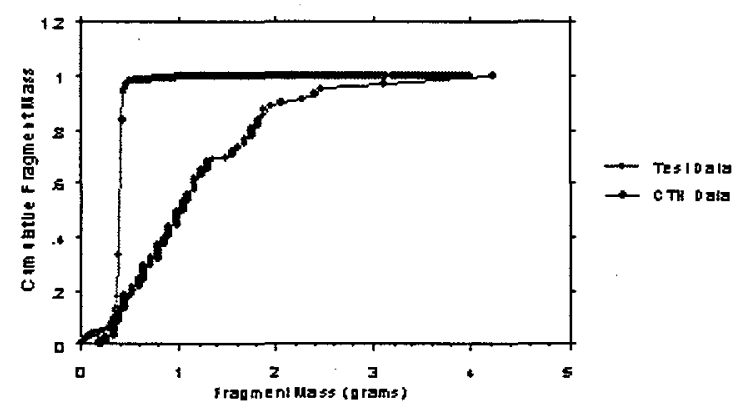

Figure 9. Comparison of Experimental and "Raw" Calculated Fragment Cumulative Mass Distributions

As can be seen, while the mean fragment size estimated from the model may compare well with that experimentally determined, the resulting cumulative distribution is clearly different. However, when a Poisson distribution is imposed on these local averages in each bin, the sums of the contributions from each bin then lead to an overall statistical distribution for the event (Grady and Kipp, 1985; Kipp, et al., 1993). Note that this does not account for the observations that there are local unequal strain rates in the tungsten. Yet, it leads to a much closer correlation to the experimentally derived distribution as is illustrated in Figure 10.

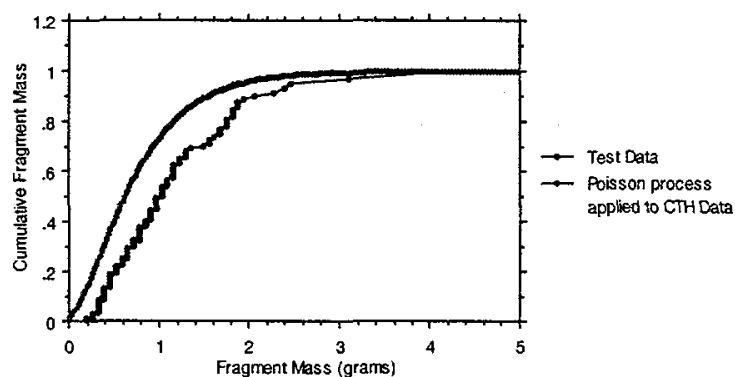

Figure 10. Comparison of Experimental and Post-processed Calculated Fragment Cumulative Mass Distributions

\subsection{The AerMet 100 Steel Experiment}

The cross-section of the axisymmetric numerical representation of the AerMet 100 steel experiment and the corresponding framing camera results are shown in Figures 11 through 13. Note that in the axisymmetric representation, pressure contours are on the left and material plots are on the right. The detonation of the explosive and expansion of the cylinder is akin to that of the $90 \%$ tungsten alloy (c.f., Figures 3 to 5). Again, the central region of the AerMet 100 steel cylinder experiences the first radial outward motion. As the curved front of the detonation wave expands along the cylinder wall, the wall assumes the curved shape seen in Figures 12 and 13 at 25 and 50us. Once again, the experimental external cylinder contours correlate well with the computational predictions. 

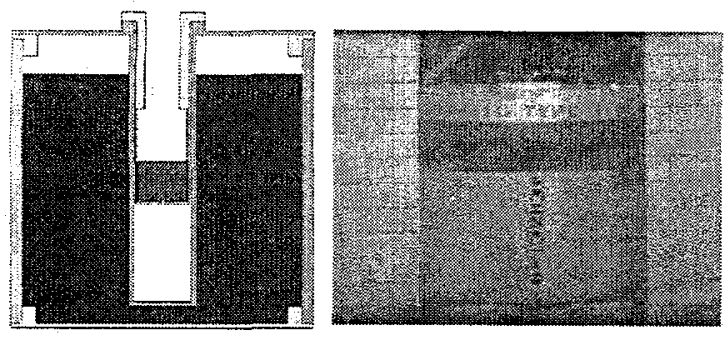

Figure 11. Comparison of CTH Calculation and AerMet 100 Steel Cylinder at $\mathrm{T}=0 \mu \mathrm{s}$
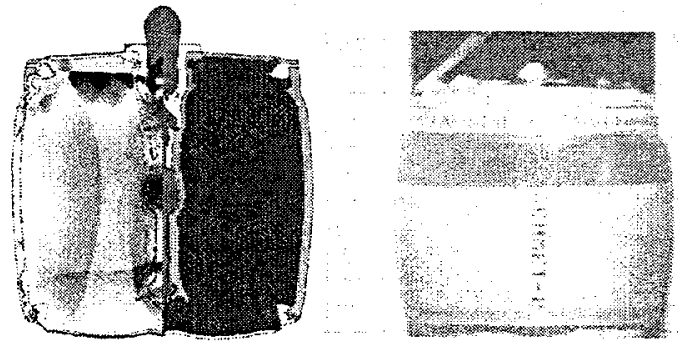

Figure 12. Comparison of CTH Calculation and AerMet 100 Steel Cylinder at $\mathrm{T}=25 \mu \mathrm{s}$
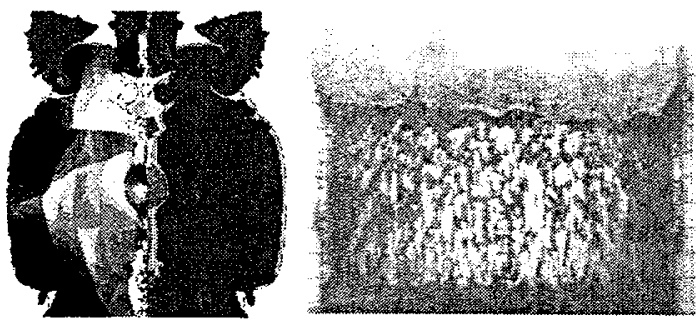

Figure 13. Comparison of CTH Calculation and AerMet 100 Steel Cylinder at $\mathrm{T}=50 \mu \mathrm{s}$

The average fragment size of the steel is considerably larger than that of the $90 \%$ tungsten alloy, primarily because of the effect of the smaller density and larger fragmentation toughness of the steel (c.f., Equation 1). This is clearly seen when one compares the results shown in Figure 7 with those of Figure 14 where the strain to failure estimated from the recovered fragments is about $30 \%$.

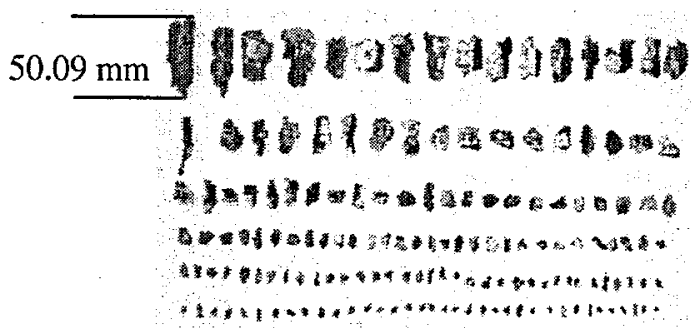

Figure 14. Fragments Recovered from the AerMet 100 Steel Test

Note that the strain rates at the time of fracture are comparable to those of the tungsten, because the radial velocities and displacements are similar in both cases. The average fragment size of about $8 \mathrm{~mm}$, indicated in the distributions (Figure 15), leads to a fragment mass of about $4.5 \mathrm{~g}$ (70 grains). The average mass value from the experimental data is 51 grains when an exponential distribution is assumed. Again, there is a disparity in circumferential and axial strain rates, leading to axial sizes of nearly $20 \mathrm{~mm}$ for an aspect ratio of 2.5. Combining this dimension with the local thickness at failure (about $7 \mathrm{~mm}$ ), and the circumferential dimension of about $8 \mathrm{~mm}$, a fragment mass of about $9 \mathrm{~g}$ (140 grains) is calculated. For these thicker steel shells, Mock and Holt (1983) found that there were many fragments that did not extend through to both the inner and outer surfaces. It is the intent of the statistical distribution associated with the local average fragment size to accommodate such contributions to the overall fragment size distribution. 

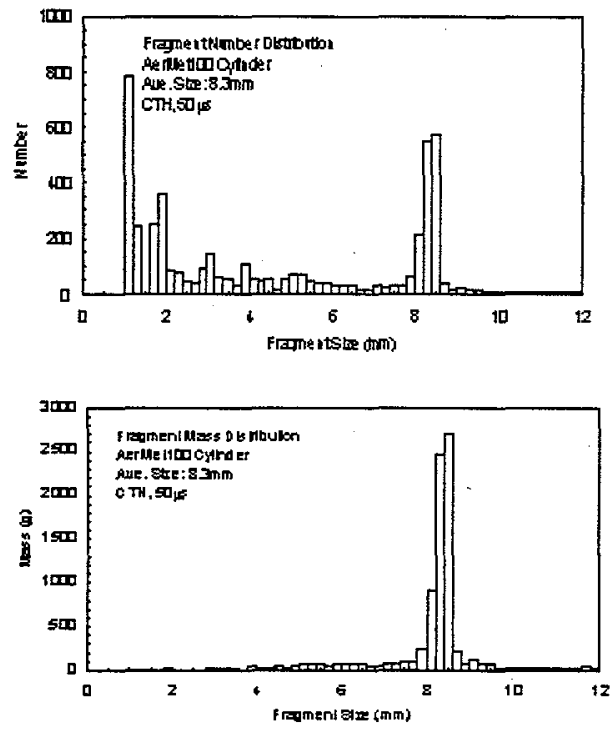

Figure 15. Fragment Number (Top) and Mass (Bottom) Distributions for theAerMet 100 Steel Cylinder (Fragment Bin Size $0.2 \mathrm{~mm}$ )

Figure 16 compares the cumulative mass distribution that is predicted using CTH with that experimentally determined. Once again, there is significant disparity in the results when only the local average fragment size is considered. As can be seen, the CTH prediction shows that most of the fragment mass is concentrated in a few fragment bins, a consequence of the narrow range of strain rates present in the cylinder. This results in a much steeper rise to the distribution that is not physically correct.

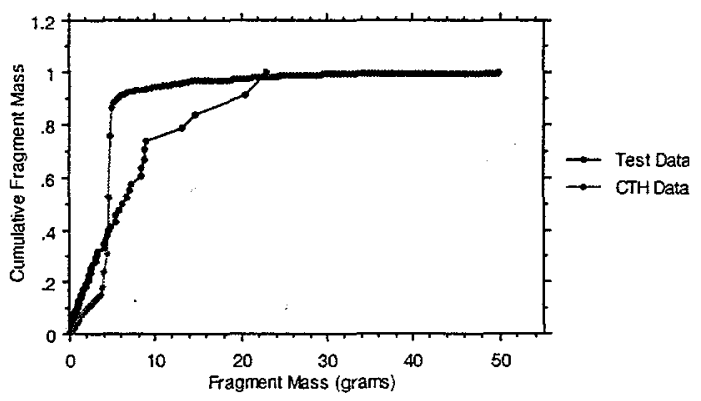

Figure 16. Experimental and "Raw" Calculated Fragment Cumulative Mass Distributions
As with the $90 \%$ tungsten cylinder, imposing a Poisson distribution on each of the fragment bins, and summing these distributions, results in much better agreement with the experimental data (Figure 17).

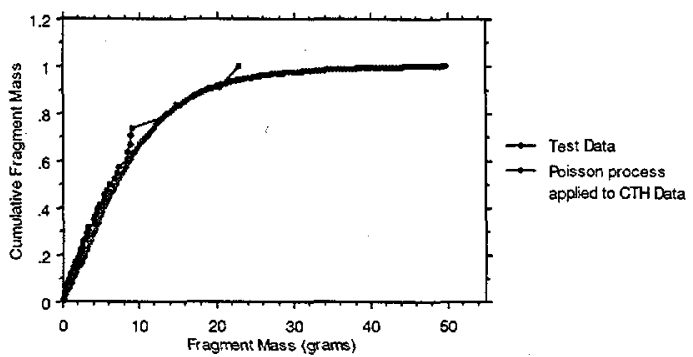

Figure 17. Experimental and Post-processed Calculated Fragment Cumulative Mass Distributions

\subsection{DISCUSSION}

The results presented indicate that the Grady-Kipp fracture and fragmentation model, as implemented into $\mathrm{CTH}$, provides reasonable estimates of average fragment mass. However, these average measures do not provide results that match the experimentally determined cumulative mass distributions. When Poisson statistics are applied to the local average fragment sizes, the agreement between the numerical and experimental results improves dramatically. This is especially encouraging, since the two materials studied are so dissimilar in nature.

In the future, we would like to improve the coupling of the Poisson statistics within the model. We would also like to accommodate multiple local strain rates and case thickness in a more inclusive way in order to determine fragment size distributions. Addressing multiple local strain rates is especially important for predicting the response of a material like steel where large "strip" fragments are generally produced. It is important to address the effects of case thickness because fragments may tend to break preferentially into sizes based upon wall thickness; and, because fragmentation occurs later in cylinders expanding under internal explosive loads than under free expansion. The internal pressure reduces the 
tensile stress, particularly on the interior of the cylinder adjacent to the explosive, and delays the formation of sufficient tensions to fracture.

These improvements would allow implementation of a physically consistent, easy to use fragmentation model into $\mathrm{CTH}$.

\section{ACKNOWLEDGMENTS}

The authors wish to recognize the following people for their work on this paper:

Dr. E. S. Hertel of Sandia National Laboratories for providing the initial interface with the Naval Surface Warfare Center Dahlgren Division (NSWCDD) in conducting these simulations.

Ms Lucia Kuhns of NSWCDD for her technical contributions on the AerMet 100 steel test data.

Ms Eva J. Dickens of Anteon Corporation for editing and preparing this paper for publication.

\section{REFERENCES}

1. Dobratz, B. M. and P. C. Crawford (1985). "LLNL Explosives Handbook. Properties of Chemical Explosives and Explosive Simulants", Lawrence Livermore National Laboratory Report, UCRL- 52997, Change 2, January 1985.

2. Grady, D. E. (1988). "The Spall Strength of Condensed Matter", J. Mech. Phys. Solids, 36, 353-384.

3. Grady, D. E. and D. A. Benson (1983). "Fragmentation of Metal Rings by Electromagnetic Loading", Experimental Mechanics, 23, 393-400.

4. Grady, D. E. and M. E. Kipp (1985). "Geometric Statistics and Dynamic Fragmentation", J. Appl. Phys., 58, 1210-1222.
5. Group GMX-6 (1969). "Selected Hugoniots", Los Alamos Scientific Laboratory Report LA4167-MS.

6. Johnson, G. R. and W. H. Cook (1985). "Fracture Characteristics of Three Metals Subjected to Various Strains, Strain Rates, Temperatures and Pressures", Engineering Fracture Mechanics, 21, 31-48.

7. Kipp, M. E. and D. E. Grady (1986). "Random Flaw Nucleation and Interaction in One Dimension", in Metallurgical Applications of Shock-Wave and High-Strain-Rate Phenomena", Editors: L. E. Murr, et al., Marcel Dekker, New York.

8. Kipp, M. E., D. E. Grady, and J. W. Swegle (1993). "Experimental and Numerical Studies of High-Velocity Impact Fragmentation", Sandia National Laboratories Report, SAND93-0773, August 1993.

9. McGlaun, J. M., S. L. Thompson, and M. G. Elrick (1990). "CTH: A Three-Dimensional Shock Wave Physics Code", Int. J. Impact Engng., 10, 351-360.

10. Mock, W. and W. H. Holt (1983). "Fragmentation Behavior of Armco Iron and HF1 Steel Explosive-Filled Cylinders", J. Appl. Phys., 54, 2344-2351.

11. Mott, N. F. (1943). "A Theory of the Fragmentation of Shells and Bombs", British Ministry of Supply Report A. C. 4035.

12. Rice, D.J., W. Kreider, C. Garnett, L.T. Wilson, (1996), "Comparing Fragmentation Characteristics of Tungsten, Tantalum, and Steel", Proceedings of the $16^{\text {th }}$ International Symposium on Ballistics, San Francisco, CA.

Sandia is a multiprogram laboratory operated by Sandia Corporation, a Lockheed Martin Company, for the United States Department of Energy under contract DE-AC04-94AL85000. 